\title{
YIELDS FROM BLOOD CULTURES OF PATIENTS WITH SUSPECTED PARATYPHOID FEVER A
}

\author{
Wang, S.k. ${ }^{12^{*}}$; Chu, C.J. ${ }^{3}$; Shan, D.S. ${ }^{3}$; Sun, P.S. ${ }^{1}$; Wu, Q. ${ }^{2}$ \\ ${ }^{1}$ College of Life Science, Yunnan University, Kunming 650091, Yunnan, China; ${ }^{2}$ Center for Disease Control and prevention of \\ Yuxi city, Yuxi 653100, China; ${ }^{3}$ People's Hospital of Yuxi City, Yuxi 653100, China.
}

Submitted: December 21, 2008; Returned to authors for corrections: May 06, 2008; Approved: November 07, 2009.

\begin{abstract}
The yield and speed of detection of Salmonella enterica serotype Paratyphi A from the blood of patients with suspected paratyphoid fever A in 13500 paired aerobic and anaerobic bottles (AEB, ANB) that were each filled with $5 \mathrm{ml}$ of blood by the BacT/ALERT 3D system were compared, and the blood bacterial counts of 1000 probable patients were estimated by pour plate method. A total of 4060 isolates were recovered, of these, 3149 were recovered from both AEB and ANB, 461 from the AEB only, and 450 from the ANB only. The estimating median bacterial count in blood from 400 patients was $0.5 \mathrm{CFU} / \mathrm{ml}$. The research findings demonstrate that the blood volume drawn is an important factor determining the yields from blood cultures. Growth of significantly more isolates was detected earlier in AEB.
\end{abstract}

Key words: Paratyphoid fever; Salmonella enterica serotype Paratyphi A; blood Culture; bacterial counts in blood

Paratyphoid fever A (PA) is a systemic infection caused by Salmonella enterica serotype Paratyphi A (SPA)(1, 3). Although blood cultures are considered the "gold standard" for the detection of bloodstream pathogens, there is little information on the controlled clinical comparison of the paired aerobic and anaerobic bottles in the blood of patients with PA $(2,4,6,7,8,9)$. Yuxi city is the most severe endemic area of PA in China (10). In order to confirm the factors determining the yields from blood cultures, blood cultures we performed by the BacT/ALERT 3D automated blood culture system (Bio Mérieus, Inc., Durham, N.C.) for a large series of patients with suspected PA between 1999 and 2008.

A total of 13500 patients admitted with suspected PA were studied. The available blood samples were obtained with vacuum system blood collection tubes. A blood culture set included one aerobic bottle (AEB) and one anaerobic bottle
(ANB) [Bio Mérieus]. A 10-ml quantity of blood from each patient for one test set was considered the maximum amount to obtain from a single venipuncture and was distributed as follows: $5 \mathrm{ml}$ to one AEB, and $5 \mathrm{ml}$ to one ANB. Each bottle was monitored with BacT/ALERT $3 \mathrm{D}$ system at $37^{\circ} \mathrm{C}$ for a standard 5-day protocol or until the instrument signaled a positive result (4). Any bottle with a positive signal was immediately removed from the instrument, and an aliquot of the blood-broth mixture was taken from the bottle with a sterile needle and swabed for subculture on sheep blood nutrient agar and XLD agar (Oxoid, England); the time-to-detection of bacteria causing PA was taken as the incubation time required before the instrument gave a positive signal. All bottles were treated independently until the end of the 7-day incubation and testing period.

All organisms isolated following subculture were

*Corresponding Author. Mailing address: Center for Disease Control and Prevention of Yuxi City, Dr. Wang Shukun. Yuxi 653100, China.; Tel.:86-8778882676 Fax: 86-877-8882615.; E-mail: yxwsk@ hotmail.com 
identified to the serotype level by agglutination tests with Salmonella O2-and Ha-specific antiserum (Biological Products Institute of the Ministry of Public Health, Lanzhou, China) and standard biochemical tests (Automatic Microorganism Identification System VITEK 32 GNI+, bioMérieux) (3, 5). For each isolate, the time-to-detection (h) of SPA isolates were recorded. Comparisons of the yields and speed of detection on SPA in blood culture bottles were evaluated statistically by using SPSS, VERSION 11.5. Statistical significance was determined when the $p$-value was less than 0.01 .

During the study period, 13500 blood cultures sets were processed in both AEB and ANB pairs. Of the sets, a total of 4 060 SPA isolates were recovered and identified. Of these, 3 $149(77.6 \%)$ grew SPA in both bottle pairs, 461 (11.3\%) grew only in AEB, and 450 (11.1\%) grew only in ANB; 3610 were recovered from $\mathrm{AEB}$ and 3599 were recovered from ANB. The total recovery rate of SPA $(10 \mathrm{ml}$ venous blood per person) by using paired culture bottles was 30.1\%. The recovery ( $5 \mathrm{ml}$ venous blood per person) by using AEB was $26.7 \%$, and the recovery by using ANB was also $26.7 \%$. No significant differences were seen between AEB and ANB in terms of recovery rate $\left(\chi^{2}=0.023 P=0.880\right)$. Significantly more SPA strains grew from both bottle pairs than from AEB only and ANB only $(P<0.01)$. The sensitivities of blood cultures with AEB and ANB alone were $88.9 \%$ and $88.6 \%\left(\chi^{2}=0.149\right.$, $P=0.700$ ), respectively. The corresponding probabilities of missed detection were $11.1 \%$ and $11.3 \%$, respectively.

Each culture was examined in order to determine which bottle gave the earliest positive result in each set. Of the 4060 SPA isolates, the growth of $484(12.0 \%)$ isolates was detected at the same time in both bottles, the growth of 1947 (47.9\%) isolates was detected first in AEB bottles, and the growth of 1 $629(40.1 \%)$ isolates was detected first in ANB bottles. Of the 3149 SPA isolates recovered from both bottle pairs, the growth of $1500(47.6 \%)$ isolates was detected first in AEB bottles and the growth of $1165(37.0 \%)$ isolates was detected first in ANB bottles $(P<0.01)$. As shown in Figures 1 and 2 , nearly all SPA isolates were recovered within the first $72 \mathrm{~h}$ of incubation and testing, 4060 SPA isolates were all recovered from AEB and ANB by the fifth day of incubation and testing, and no isolate was recovered on the sixth day or more. For 3 149 blood culture sets in which both bottles grew SPA, the mean times required to detect SPA growth were $23.66 \pm 15.89$ and 25.48 $\pm 16.92 \mathrm{~h}$ for AEB and ANB, respectively $(\mathrm{t}=7.007, P$ $<0.01)$. The AEB time for 461 isolates was $32.85 \pm 23.33 \mathrm{~h}$, while the ANB time for 450 isolates was $34.46 \pm 18.44 \mathrm{~h}(\mathrm{t}=$ $3.2, P<0.01)$. Overall, there was a significant difference in the number of cultures detected earlier between AEB and ANB; growth was more often detected earlier in AEB than in ANB for SPA $(\mathrm{t}=3.0, P<0.01)$.

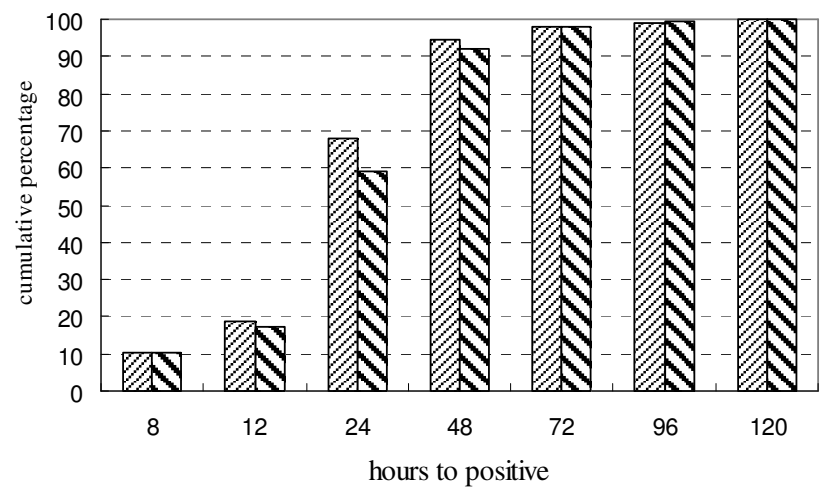

Figure 1. Cumulative percentages of group blood culture bottles that grew SPA isolates.

aerobic bottle; $\mathbf{N}_{\text {anaerobic bottle; }}$

In the study reported here, the performances of AEB and ANB in the BacT/ALERT system showed substantial comparability in both yield and time to the detection of positive blood cultures in patients with suspected PA. With the exception of the mean time to positive blood culture bottles, which was detected earlier more frequently in AEB, there were no significant differences in terms of SPA recovery between the two bottles. As paired bottles in a blood culture set, AEB and ANB would provide excellent recovery of SPA from PA patients. AEB was shown to be superior to ANB for the recovery of SPA; the AEB procedure is more appropriate for the blood culture of PA patients because it results in the shortest time-to-detection for SPA. 


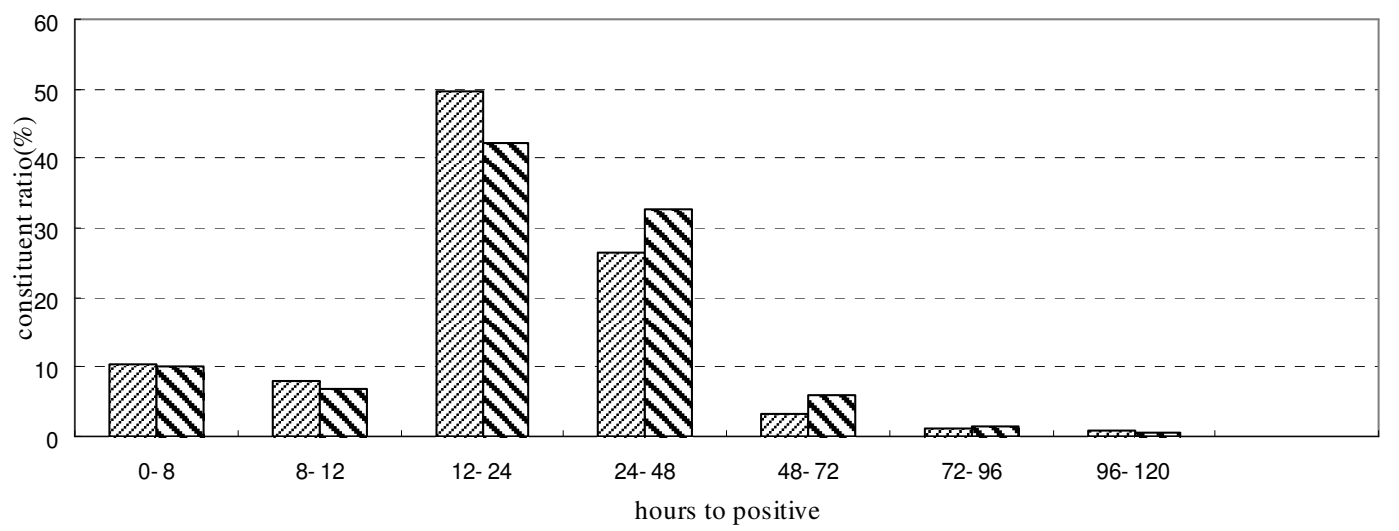

Figure 2. The constituent ratio of blood culture bottles that grew SPA isolates at the indicated time interval.

\author{
aerobic bottle; $\mathbf{N}_{\text {anaerobic bottle; }}$
}

Data from the present study confirm those from an earlier study showing that standard bottles can be incubated and tested for 5 days without a significant decrease in the recovery of clinically important microorganisms (4). The study also provides formal mathematical proofs on the concentration of SPA in the blood, which the probability of a positive blood culture, in part, depends on the volume of sample processed. In the past, several studies have demonstrated enhanced recovery of bacteria in blood cultures for septicemias when using a twobottle blood culture set due to the additional blood volume, and all have shown the sensitivity of the culture procedure to be volume-dependent $(2,8,9)$. In this study, exactly the same pattern was observed, 4060 clinically SPA isolates were all recovered from AEB and ANB by a standard 5-day protocol. In conclusion, all of findings illustrate that the volume of blood taken is an important factor determining the yields from blood cultures.

\section{REFERENCES}

1. Bhan, M.K.; Bahl, R.; Bhatnagar, S. (2005). Typhoid and paratyphoid fever. The Lancet, 366(9478): 749-762.

2. Cockerill, F.R.; Wilson, J.W.; Vetter, E.A.; Goodman, K.M.; Torgerson, C.A.; Harmsen, W.S.; Schlerk, C.D.; Iistrup, D.M.; Washington, II. J.A.; Wilson, W.R. (2004). Optimal testing parameters for bloodcultures. Clin. Infect. Dis., 38(6): 1724-1730.
3. Costalunga; Suzana and Tondo; Eduardo Cesar. Salmonellosis in Rio Grande do sul, Brazil, 1997 to 1999 Braz. J. Microbiol., 2002, 33(4):342346.

4. Hardy, D.J.; Hulbert, B.B.; Migneault, P.C. (1992). Time to detection of positive BacT/Alert blood cultures and lack of need for routine subculture of 5- to 7-day negative cultures. J. Clin. Microbiol., 30(10): 2743-2745.

5. Hendriksen RS: A global Salmonella surveillance and laboratory support project of the World Health Organization: Laboratory Protocols Level 1 Training Course Identification of Salmonella $4^{\text {th }}$ Ed. April 2003. http://www.antimicrobial resistance. dk/data/images/salmonella2-pdf.pdf

6. Lee, A.; Stanley, M.; Reller, L.B.; Weinstein, M.P. (2007). Detection of bloodstream infections in adults: how many blood cultures are needed? $J$. Clin. Microbiol., 45(11): 3546-3548.

7. Marconi, C.; Cunha, M.L.R.S.; Lyra, J.C.; Bentlin, M.R.; Batalha, J.E.N.; Sugizaki, M.F.; Rugolo, L.M. (2008). Comparison between qualitative and semiquantitave cather-tip cultures: laboratory diagnosis of cathetertip cultures-related infevtion in newborns. Braz. J. Microbiol., 39(2): 262-267.

8. Riedel, S.; Bourbeau, P.; Swartz, B.; Brecher, S.; Carroll, K.C.; Stamper, P.D.; Dunne, W.M.; McCardle, T.; Walk, N.; Fiebelkorn, K.; Sewell, D.; Richter, S.S.; Beekmann, S.; Doern, G.V. (2008). Timing of specimen collection for blood cultures from febrile patients with bacteremia. $J$. Clin. Microbiol., 46(4): 1381-1385.

9. Wain, J.; Diep, T.S.; Ho, V.A.; Walsh, A.M.; Hoa, N.T.T.; Parry, C.M.; White, N.J. (1998). Quantitation of bacteria in blood of typhoid fever patients and relationship between counts and clinical features, transmissibility, and antibiotic resistance. J. Clin. Microbiol., 36(6): 1683-1687.

10. Zhang, H.Q.; Wang, S.K.; Wang, Y.F. (2008). Epidemic analysis of typhoid and paratyphoid fever A from 1998 through 2004 in Hongta area of Yuxi city. Chinese Journal of Natural Medicine (Chin)., 10 (6): 401405 . 